\title{
Tecnura
}

http://revistas.udistrital.edu.co/ojs/index.php/Tecnura/issue/view/640

DOI: http://dx.doi.org/10.14483/udistrital.jour.tecnura.2014.SE1.a12

REVISIÓN

\section{Aplicación de los sistemas fotovoltaicos conectados a la red: estado del arte}

\section{Grid-connected photovoltaic systems application: state of the art}

\author{
Johann Alexander Hernández Mora*, Andrés Felipe Cortés Borray** \\ Daniel Alberto Balaguera Cañola***, Mauricio Alexander Urueña Saavedra***
}

Citation / Para citar este artículo: Hernández Mora, J. A., Cortés Borray, A. F., Balaguera Cañola, D. A., \& Urueña Saavedra, M. A. (2014). Aplicación de los sistemas fotovoltaicos conectados a la red: estado del arte. Revista Tecnura, Edición especial, 157-172.

Fecha de recepción: 14 de julio de 2013 / Fecha de aceptación: 16 de mayo de 2014

\section{Resumen}

En este trabajo se describen todos los conceptos necesarios para lograr implementar un sistema fotovoltaico como alternativa para ser utilizado como generador distribuido. Para ello, se realiza un pequeño estado del arte en el cual se manejan conceptos básicos, desde el funcionamiento de una celda solar hasta conceptos de redes inteligentes aplicados en la gestión de la demanda. Asimismo, se abarca un repaso sobre los marcos regulatorios existentes en Colombia, donde se habla de generación distribuida debido a la falta del mismo. Es necesario comparar tales regulaciones con los diferentes marcos regulatorios existentes en otros países, como Alemania, España y EE.UU., para obtener un modelo de trabajo idóneo. Finalmente, se decide utilizar un generador fotovoltaico conectado a la red de baja tensión como alternativa en los sistemas de generación distribuida, ya que con estos se pueden realizar balances energéticos, esto en caso de que en el país en donde se emplee esta alternativa no se tenga establecido un marco regulatorio adecuado, de manera que el usuario no tenga problemas con las entidades regulatorias actuales.

Palabras clave: balance energético, energías renovables, generación distribuida, gestión de la demanda, sistemas fotovoltaicos.

\begin{abstract}
This paper outlines all necessary concepts to successfully implement a photovoltaic system as an alternative in a distributed generation scheme. For this purpose, it is made a short state of the art in which basic concepts are addressed, from a solar cell operation to smart grid concepts applied on demand management. Likewise, a review of the different regulatory frameworks in Colombia is entailed, especially those related to distributed generation, due to the lack of these ones. It is necessary to make a comparison with other regulations from other countries,
\end{abstract}

\footnotetext{
* Ingeniero electricista, magíster en ingeniería eléctrica con énfasis en distribución; doctor en ingeniería eléctrica. Docente, Universidad Distrital Francisco José de Caldas. Bogotá, Colombia. Contacto: jahernandezm@udistrital.edu.co

** Ingeniero eléctrico, estudiante de maestría en integración de las energías renovables. Universidad del País Vasco. Bilbao, Vizcaya. España. Contacto: acortes024@ikasle.ehu.es

*** Ingeniero eléctrico. Jefe del área de ingeniería, ENERSA S.A. Proyectos en Energías Renovables y Eficiencia Energética. Bogotá, Colombia. Contacto: dabalaguerac@correo.udistrital.edu.co

**** Ingeniero eléctrico. Ingeniero de soporte de mantenimiento, FYR Ingenieros. Bogotá, Colombia. Contacto: mauruenas@correo.udistrital. edu.co
} 
such as Germany, Spain and USA, in order to obtain a suitable work model. Finally, a photovoltaic system connected into the low voltage grid is used as an alternative in distributed generation systems, due to the fact that with this system energy balances can be performed, in case that countries where this alternative is implemented may not have an adequate regulatory framework established, so that users will not have problems with regulatory entities.

Keywords: demand management, distributed generation, energy balance, photovoltaic systems, renewable energies.

\section{INTRODUCCIÓN}

El desarrollo de la energía eléctrica es uno de los procesos de innovación que más han impactado en el progreso de la humanidad y para constatarlo, solo se debe echar un vistazo a nuestro alrededor y percibir cómo todo lo que nos rodea requirió en su desarrollo y su funcionamiento el uso de energía eléctrica.

Desde su aparición, la energía eléctrica ha requerido un proceso de generación y distribución, siendo esta la base del sistema, afectando no solo el bienestar de las personas sino también la economía de las naciones. Sin embargo, las potencias tecnológicamente más avanzadas se han preocupado por garantizar la oferta energética en el mundo, investigando y desarrollando nuevas tecnologías en cuanto a fuentes no convencionales de producción de energía eléctrica (Gischler \& Janson, Noviembre 2011) y (Casmus \& Eusebio, 2008), puesto que estas reducen parcialmente la cantidad de elementos contaminantes en comparación con los sistemas de combustibles fósiles, los cuales durante las últimas décadas han contribuido a la emisión de millones de toneladas de $\mathrm{CO}_{2}$ (Grundi, 2008).

Por ello, hoy día las investigaciones han puesto la mirada en el uso de fuentes limpias de generación, además de implementar diferentes políticas que justifiquen este interés, como lo indican estudios hechos por el REN21 (Renewable Energy Police Network for the 21st Century). Según los reportes del REN21, actualizados al año 2013, la contribución de las fuentes de energía no convencionales a la producción de energía eléctrica mundial es de $16 \%$, donde la generación fotovoltaica contribuye con $0.6 \%$ de dicha producción (REN21, 2012). Por tanto, este tipo de tecnologías podrán ser utilizadas en los sistemas de transmisión y distribución de energía eléctrica, lo cual mitigará las grandes pérdidas e inconvenientes de confiabilidad que tienen los sistemas de potencia hoy día (Singh \& Parida, 2012).

Los recientes avances obtenidos en el campo de la generación a pequeña escala y almacenamiento eléctrico implican cambios en la forma en que se ha ido concibiendo la generación y distribución de energía en las últimas décadas, donde se retoma con vigor el concepto de generación distribuida (GD) (Wijesinghe \& Lei Lai, 2011).

Para lograr que el concepto de generación distribuida comience a tener gran auge a nivel mundial es necesario que este garantice aspectos como eficiencia, confiabilidad y calidad en el servicio prestado de energía eléctrica. En extensos trabajos, diversos investigadores (Gischler \& Janson, Noviembre 2011) y (Strachan \& Dowlatabadi, 2002) discuten acerca de las ventajas de la GD en aspectos de eficiencia, flexibilidad, interconexión con redes de distribución, niveles de emisión de gases de las plantas de generación aisladas, e inversiones por instalación y costos de mantenimiento y funcionamiento (Strachan \& Dowlatabadi, 2002), en donde se resaltan generalmente aspectos que identifican la GD como una alternativa con grandes proyecciones para diversos usuarios, que demandan consumos inferiores a $10 \mathrm{MW}$ y/o ciertos requerimientos especiales de generación y distribución.

Las tecnologías de generación distribuida se dividen, a su vez, en convencionales y no 
convencionales. Las primeras incluyen motores de combustión interna, microturbinas y turbinas a gas, mientras que las segundas se refieren a la implementación de $\mathrm{PCH}$, turbinas eólicas (motores síncronos o de inducción), geotérmica, biomasa y módulos fotovoltaicos. Por tanto, para que este tipo de tecnologías ofrezca mayor confiabilidad al sistema es factible utilizar almacenadores de energía, puesto que en la mayoría de los casos no es posible controlar el recurso energético (Bayod, 2012). Entre las tecnologías de almacenamiento se encuentran los acumuladores químicos (baterías), volantes de inercia (flywheels), bobinas superconductoras (SNER), celdas de combustible hidrógeno y súper capacitores (Hernández, 2012).

Actualmente en Colombia se presentan casos que pueden enmarcarse dentro del concepto de generación distribuida, entre estos casos se destacan proyectos existentes como el de energía eólica a escala media y de energía solar a baja escala (Bayod, 2012), tomando como punto de partida lo mencionado en el objetivo del plan de desarroIlo 2011-2030 para fuentes no convencionales de energía (FNCE) (Consorcio Energético CORPOEMA, Diciembre, 2010). Esto permite planear nuevas estrategias en aquellas áreas en donde se tenga el potencial necesario para lograr la implementación de este tipo de tecnologías de generación y almacenamiento y así, lograr mayor cobertura a niveles residencial, comercial e industrial en el contexto nacional colombiano.

En tal sentido, el enfoque que tiene esta investigación radica en el uso de tecnologías no convencionales para generación distribuida, utilizando principalmente módulos fotovoltaicos para su desarrollo. Conforme al uso histórico que han tenido los sistemas fotovoltaicos en Colombia, este generalmente está relacionado con su implementación en zonas apartadas o con difícil acceso al Sistema de Interconexión Nacional (SIN) debido a su alto costo de instalación (Rodríguez, Noviembre, 2008), por lo cual se plantea la idea de empezar a relacionar este tipo de tecnología dentro del SIN.
Lograr la propagación del concepto de generación distribuida mediante la aplicación de sistemas fotovoltaicos conlleva grandes retos relacionados con la planeación, inversión, operación y regulación de los sistemas eléctricos tradicionales. Para los sistemas interconectados, el usuario final podría llegar a entregar energía eléctrica al sistema en el lado de baja tensión, lo que permitiría reducir las pérdidas de transporte y distribución, al igual que incrementar la extensión del sistema y disminuir la dependencia de los sistemas centralizados. Por tal razón, observando el contexto colombiano, es necesario identificar las condiciones técnicas de interconexión para estos nuevos generadores, de tal forma que se mantengan los niveles de confiabilidad establecidos por la CREG 070 de 1998 (CREG, Comisión Reguladora de Energía y Gas, Mayo, 1998).

Los sistemas fotovoltaicos interconectados a la red en países como Alemania y España están siendo utilizados como complemento a los sistemas de generación centralizada (Caamaño, 1998). La generación distribuida ha sido pieza fundamental para que este tipo de tecnología alcance un grado de importancia para que empiece a ser implementada en áreas urbanas, donde las plantas de pequeña capacidad de generación son instaladas en los techos de edificios o residencias dentro del concepto "Photovoltaic Grid Connected Buildings" (PVGCB) (Caamaño, 1998).

\section{SISTEMA FOTOVOLTAICO}

Un sistema fotovoltaico está constituido por una agrupación de módulos y un conjunto de elementos que adaptan la energía eléctrica que produce el generador a la aplicación (Caamaño, 1998; Fathpour, Tsia, \& Jalali, 2007; Gerol \& Neudck, 1990). Este sistema se puede clasificar en dos grandes grupos según su aplicación: sistemas autónomos y sistemas interconectados a la red eléctrica. En la figura 1 se muestra el diagrama de bloques para las dos clasificaciones. 
El primer grupo requiere asegurar la disponibilidad de electricidad aun en los casos en donde la generación es inferior a la demanda o cuando no está visible el sol, con lo cual no hay generación. Para ello es indispensable almacenar la energía que se produce durante los períodos en que la generación supera a la demanda (Zuñiga \& Botina, 2012).

En el segundo grupo no existen requerimientos de seguridad en el suministro de energía, de modo que los criterios para la elección de la potencia del generador son más ambiguos, en este caso la red es la fuente infinita de energía eléctrica. Este tipo de sistemas permite intercambiar energía eléctrica con la red cuando la generación excede las necesidades de energía del usuario y tomar energía de la red cuando la demanda es mayor que la energía generada por el arreglo fotovoltaico (Assi, Jama, \& Al Kathairi, 2009).

\section{SISTEMA FOTOVOLTAICO CONECTADO A LA RED (SFVCR)}

Un sistema fotovoltaico interconectado a la red consiste en un arreglo de elementos, entre los cuales están los módulos solares e inversores. El objetivo de este tipo de tecnología consiste en generar energía eléctrica a partir de radiación primaria (solar). Mediante el efecto fotovoltaico, los paneles solares transforman la energía proveniente de los fotones que inciden sobre el material, en un flujo de electrones que se comportan como una fuente de corriente directa (Fathpour, Tsia, \& Jalali, 2007). Al ser conectados en instalaciones en donde la tensión de la red es de naturaleza alterna se hace necesario utilizar un inversor que permita inyectar al sistema de potencia electricidad desde el generador fotovoltaico.

La figura 2 muestra un diagrama de bloques específico para un SFVCR, donde se encuentran cada uno de los componentes que hacen parte de este y que pueden ser aplicados en la GD. Los seis bloques funcionales que lo conforman se describen a continuación.

- Generador fotovoltaico: formado por los módulos fotovoltaicos y su correspondiente estructura de soporte.

- Acondicionador de potencia: es el responsable de adecuar los parámetros de potencia producidos por el generador DC a tensión variable, a

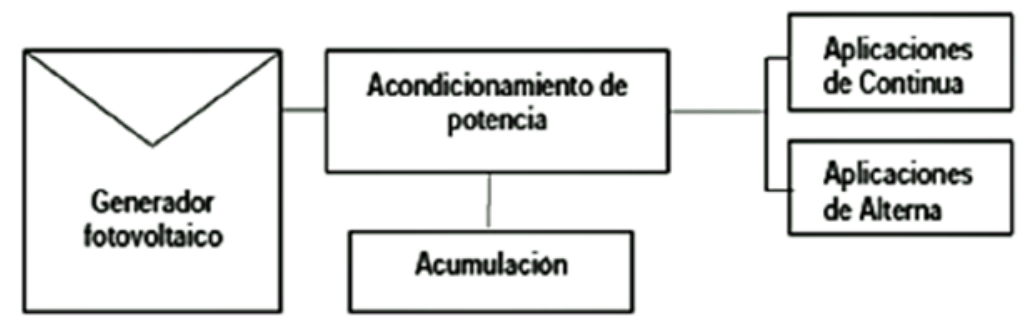

(a)

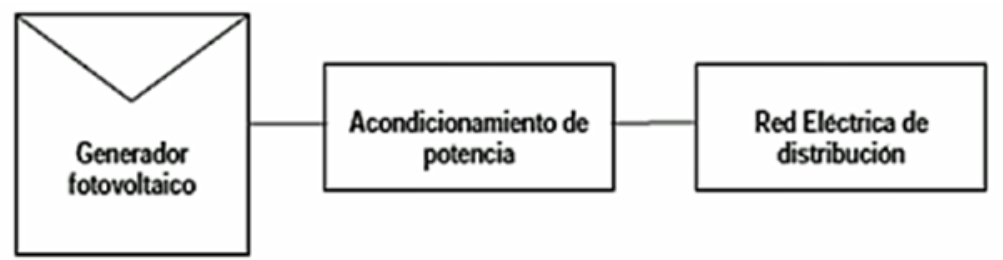

(b)

Figura 1. Diagrama de bloques (a) Sistema autónomo, (b) Sistema interconectado. 


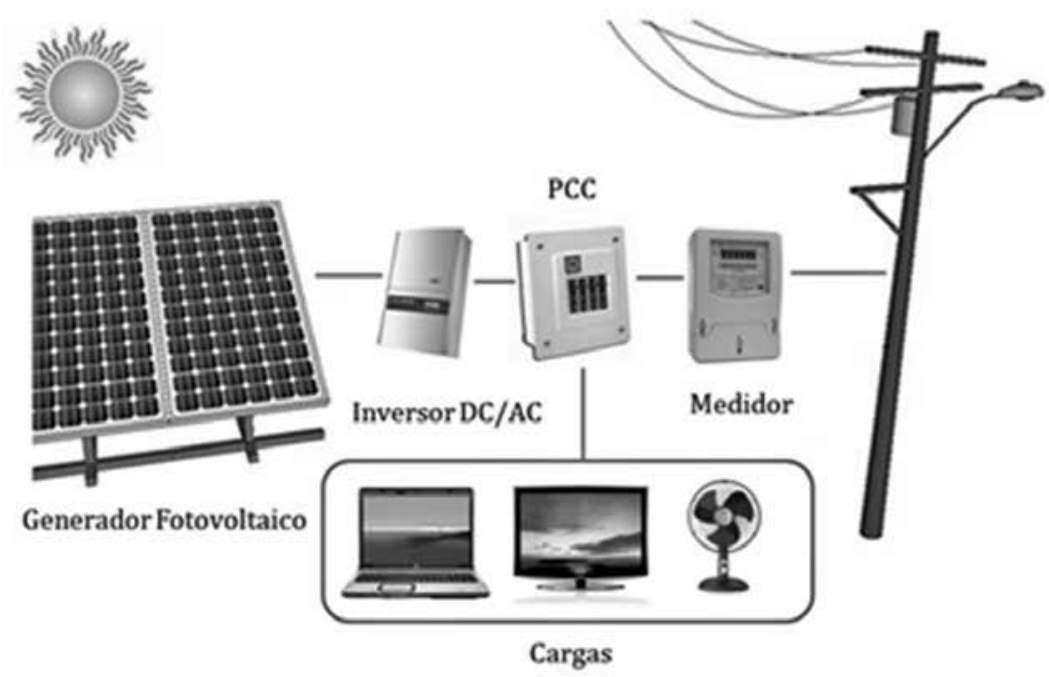

Figura 2. Sistema fotovoltaico conectado a la red.

los parámetros requeridos por la red eléctrica de baja tensión (AC a 120 o 208 V), también conocido como inversor.

- Punto de acople común: (PCC, point of common coupling), donde se ubican los elementos de protección adoptados para garantizar la seguridad del propio SFVCR y la seguridad de la red eléctrica.

- Contador bidireccional de energía AC: tiene como objeto registrar la energía que se consume de la red además de la energía que está siendo entregada al sistema interconectado en un instante de tiempo específico.

- Carga: conformada por todos aquellos elementos (lineales y/o no lineales) que demandan energía eléctrica para su funcionamiento.

- Red eléctrica.

Su funcionamiento con la red de energía eléctrica consiste en la transformación de la energía DC producida por el generador fotovoltaico en corriente alterna; esta corriente debe garantizar los mismos parámetros de la red, para así poder interconectarse automáticamente con esta. El inversor es el encargado de realizar este proceso, proporcionando tensión de salida estable y corriente variable en función de la irradiancia solar. La corriente alterna generada por el inversor es sincronizada con la frecuencia de la corriente de la red $(60 \mathrm{~Hz})$, posteriormente pasa por el medidor y es inyectada a la red.

\section{GENERACIÓN DISTRIBUIDA (GD)}

Cuando se hace una revisión de la literatura, en realidad no existe una definición única y común acerca del concepto de generación distribuida y muchos autores manejan diferentes esquemas, así como intervalos de trabajo, para caracterizar servicios que pueden caer en el margen de la generación distribuida (GD). Ejemplos de ello pueden ser los países angloamericanos, los cuales le Ilaman a esto "generación embebida"; en Norteamérica le llaman "generación dispersa", y en Europa y algunas partes de Asia se utiliza el término "generación descentralizada" (CIRED, 1999).

Adicionalmente, la GD puede ser clasificada de acuerdo con el rango de la unidad de la potencia de generación. El Instituto de Investigación de Potencia Eléctrica (EPRI, por sus siglas en inglés, Electric Power Research Institute), define la GD desde unos pocos kW hasta 50 MW (EPRI, 2013). De acuerdo con el Instituto de Investigación de 
Gas, esta generación típicamente se encuentra entre pocos kW y 25 MW (Gas Research Institute, 1998). Otros autores (Sharma \& Bartels, 1998; Cardell \& Tabors, 1998) definen el tamaño de la planta entre $500 \mathrm{~kW}$ y valores superiores hasta $100 \mathrm{MW}$. EI CIGRE (International Conference on Large High Voltage Electric Systems) las define como plantas pequeñas de 50-100 MW (CIGRE, 1998). Asimismo, diferentes autores (Ackerman, Andersson, \& Söder, 2001) proponen una clasificación a las unidades de GD según su capacidad:

- Micro $1 \mathrm{~W} \leq 5 \mathrm{~kW}$

- Pequeña $5 \mathrm{~kW} \leq 5 \mathrm{MW}$

- Mediana $5 \mathrm{MW} \leq 50 \mathrm{MW}$

- Grande $50 \mathrm{MW} \leq 300 \mathrm{MW}$

Sin embargo, en el contexto del presente documento se entenderá por generación distribuida el proceso de producción (o generación) y distribución de energía eléctrica a pequeña o mediana escala (desde cientos de KW hasta $10 \mathrm{MW}$ ), con una cercanía a los centros finales de consumo y con posibilidad de interactuar con las redes de interconexión eléctrica (Fathpour, Tsia, \& Jalali, 2007).

Debe aclararse, sin embargo, que el concepto de generación distribuida no es un nuevo paradigma que haya sido formulado en los últimos años, pues en realidad se trata de un concepto básico que ha cobrado nuevo impulso, alentado por diversos factores tecnológicos, así como por condiciones del mercado y políticas energéticas y ambientales (Pepermansa, Driesenb, Haeseldonckxc, Belmansc, \& D'haeseleerc, 2005).

Los esquemas comerciales de venta de energía en el mundo comenzaron a optar por sistemas de electricidad apenas a mediados del siglo XIX, y aun así hicieron falta unos años más para tener opciones reales de sistemas de generación y comercialización similares a los que posteriormente consolidaron la energía eléctrica como una de las principales fuentes de energía, con su concepto de transporte a sitios distantes de las fuentes de generación (International Energy Agency, 2002).
Antes de esto, la mayoría de los sistemas de generación eran, de hecho, sistemas de generación distribuida. Los sistemas basados en vapor, carbón, gas o energía hidráulica suministraban la energía en lugares cercanos o en el sitio de carga, en donde se hacía el consumo directo.

Resulta necesario adicionar a la definición de GD algunos elementos más que marcan la esencia fundamental de esta alternativa de generación, como el empleo de diversas fuentes de energía, como pueden ser la energía eólica, solar, térmica, hidráulica, entre otras; diferentes tipos de combustibles, como biomasa, gas natural, dísel, biodísel, etanol, gasolina, etc., en el caso de tecnologías basadas en sistemas de combustión; la alta eficiencia en la generación (normalmente asociada a procesos de cogeneración; Combined Heat and Power CHP); los altos niveles de confiabilidad en el suministro y de calidad en la energía entregada; y los relativamente bajos niveles de inversión inicial en los proyectos de generación (Jurado, Cano, \& Carpio, 2004).

En la GD se pueden identificar cuatro tipos de aplicaciones básicas definidas como: generación para carga base, generación para carga pico, generación aislada y generación para soporte de la red de distribución (A, Ackerman, Andersson, \& Söder, 2001).

El primer escenario ubica la GD como un sistema de generación principal y continua, interconectada a la red de distribución para operaciones de compra y venta de energía. El segundo escenario muestra la GD como un sistema alterno de respaldo para reducir el consumo desde la red de distribución en los períodos de mayor precio del kWh, de acuerdo con las fluctuaciones de la oferta en el mercado. El tercer caso sitúa la GD como un sistema de generación para poblaciones totalmente aisladas del sistema de interconexión eléctrica. Finalmente, el cuarto escenario sitúa la GD como un sistema de respaldo empleado especialmente en empresas de alto consumo energético solo en ciertos períodos del año, o para casos en los que se requiere elevar los niveles de confiabilidad en el suministro eléctrico (Ackerman, Andersson, \& Söder, 2001). 


\section{GD en Colombia}

El avance de la generación distribuida en Colombia ha sido bastante reducido frente al potencial de su expansión. Aunque en los Planes de Desarrollo y en el Plan Energético Nacional se presentan lineamientos para apoyar la utilización de fuentes nuevas y renovables de energía (Ochoa, 2002), existen pocas metas e incentivos, como las metas del PROURE, establecidas en la Resolución 180919/2010, para trabajar en la capacidad instalada o en la producción de energía en el país.

Las plantas de generación distribuida han surgido por iniciativa de algunos industriales y regiones localizadas en zonas aisladas, en donde no hay otra opción distinta al diésel como generación centralizada. En la tabla 1 se observa la capacidad instalada en Zonas No Interconectadas (ZNI), lo cual justifica el uso de GD en las regiones del territorio colombiano.

Tabla 1. Capacidad instalada de generación en las ZNI.

\begin{tabular}{ll}
\hline Potencia instalada & $118 \mathrm{MW}$ \\
\hline Generación diésel & $92 \%$ \\
\hline Generación con FNCE & $8 \%$
\end{tabular}

Fuente: (Consorcio Energético CORPOEMA, Diciembre, 2010)

Tabla 2. Capacidad instalada de GD en Europa.
En las ZNI las energías renovables (FNCE) son esencialmente sistemas fotovoltaicos en aplicaciones aisladas (principalmente telecomunicación). El IPSE (Instituto de Planeación y Promoción de Soluciones Energéticas) está desarrollando varios sistemas demostrativos, como la poli-generación de Nazaret (diésel + solar de 100 kW + eólica de 200 $\mathrm{kW}$ ), el proyecto en isla fuerte (sistema fotovoltaico con gas propano licuado), y un gasífero en Necoclí $(40$ kW) (E.E.P.P.M, Febrero, 2008). Adicionalmente, dentro de la fase de contratos está un sistema de generación eólico de 7 MW y una planta para el procesamiento de desechos sólidos con una capacidad de generación de 1 MW en San Andrés (Murillo \& Roldan, 2012).

\section{GD en Europa}

Recientemente el cambio climático a nivel mundial ha llevado a que el continente europeo desarrolle políticas para afrontarlo. Por ello, los sistemas de generación y distribución han mejorado la seguridad y la confiabilidad, lo cual aporta al desarrollo y crecimiento de la GD al continente.

El crecimiento de la GD es un componente integral de la "Red Inteligente Europea", la cual es efectiva y altamente sensible, por lo cual todas las partes interesadas deben estar integradas (Ruiz, Colmenar, Gil, \& Molina, Mayo 2013).

\begin{tabular}{lcccc}
\hline \multicolumn{1}{c}{ País (2010) } & Potencia instalada (GW) & COGEN (GW) & PV (MWp) & Eólico (MW) \\
\hline Alemania & 116 & 19 & 1600 & 18400 \\
\hline Reino Unido & 69 & 6,6 & 10 & 1350 \\
\hline Dinamarca & 13,6 & 4,9 & 36 & 3100 \\
\hline España & 54 & 8,1 & 15 & 10000 \\
\hline Francia & 115 & 3 & 2 & 750 \\
\hline Polonia & 34,5 & 5 & 15 & 70 \\
\hline
\end{tabular}

Fuente: (Meyer, Banberger, \& Bel, 2010). 
El continente europeo tiene problemas con recursos energéticos como el agua, la cual es escasa, y esto hace que algunos países (Lopes Ferreira, Costescu, L'Abbate, Minneo, \& Fulli, 2011) se vean en la necesidad de buscar nuevas alternativas empleando recursos energéticos no convencionales y renovables, como energía solar, eólica, cogeneración, entre otras. En la tabla 2 se resume de modo general la capacidad instalada en Europa de dichas fuentes, que son utilizadas como GD.

Asimismo, se han puesto en marcha diversas estrategias regulatorias para la promoción de las estrategias renovables, las cuales son (Fundación de la Energía de la comunidad de Madrid, 2012):

- Si la intervención regulatoria actúa sobre el precio o sobre la capacidad a instalar.

- Si la intervención actúa sobre la inversión o sobre la generación.

- Si los fondos para financiar la electricidad de origen renovable provienen de los consumidores finales o de los contribuyentes.

Estas estrategias se muestran a cabalidad en la tabla 3 .

\section{GD en Italia}

En Italia, lo que más predomina en GD son los sistemas fotovoltaicos. Estos han experimentado una tasa de crecimiento anual cercana a $700 \%$ entre el año 2007 y 2009 (Aste, Adhikari, \& Del Pero, 2011). Debido a este crecimiento surgieron diferentes problemas técnicos, como, por ejemplo, que las redes no estaban en capacidad de soportar la potencia instalada que brindaban los nuevos generadores fotovoltaicos (sur de Italia, $70 \mathrm{GW}$ ) (Aste, Adhikari, \& Del Pero, 2011). Esto llevó a la creación de una nueva norma técnica denominada ARG/elt 125/10, la cual dentro de su marco regulatorio estableció "zonas críticas" en donde se estableció los períodos de inicio y de finalización de la construcción de los generadores.

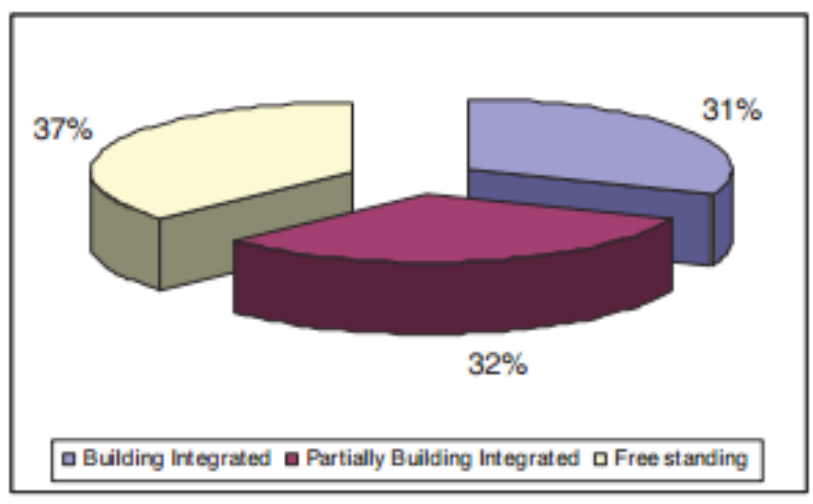

Figura 3. Integración de sistemas fotovoltaicos en edificios.

Fuente: (IEA, 2003).

Tabla 3. Estrategias regulatorias de promoción de las energías renovables.

\begin{tabular}{|c|c|c|c|c|}
\hline & & \multicolumn{2}{|c|}{ Directos } & \multirow{2}{*}{ Indirectos } \\
\hline & & Precio & Cuota & \\
\hline \multirow{4}{*}{ Regulados } & \multirow{2}{*}{ Inversión } & Ayudas a la inversión & \multirow{4}{*}{$\begin{array}{l}\text { Certificado verde } \\
\text { negociable TGC }\end{array}$} & \multirow{4}{*}{$\begin{array}{l}\text { Impuesto medioam- } \\
\text { biental }\end{array}$} \\
\hline & & Incentivos fiscales & & \\
\hline & \multirow{2}{*}{ Generación } & Primas fijas & & \\
\hline & & Subastas & & \\
\hline \multirow{2}{*}{ Voluntarios } & Inversión & Fondos verdes & & \multirow{2}{*}{ Acuerdos Voluntarios } \\
\hline & Generación & Precios verdes & & \\
\hline
\end{tabular}

Fuente: (Fundación de la Energía de la comunidad de Madrid, 2012). 
Durante los últimos años, alrededor de 4000 MWp de nuevos generadores fotovoltaicos han sido instalados en Italia (GSE, 2011); gran parte de estos se encuentran en edificaciones y algunos de ellos en zonas rurales. En la figura 3 se observa la distribución de los sistemas fotovoltaicos instalados en Italia durante los últimos años, de acuerdo con los niveles de integración de sistemas fotovoltaicos en edificios.

Con la instalación de estas plantas generadoras se estima reducir $5 \mathrm{Mt}$ de $\mathrm{CO}_{2} / a n ̃ o$, considerando un promedio de factor de emisión del parque de generación nacional igual a $0,053 \mathrm{~kg}$ de $\mathrm{CO}_{2}$ por kWh (EPIA, 2010).

\section{GD en Alemania}

Diferentes estrategias que han incrementado la capacidad de los sistemas fotovoltaicos conectados a la red de baja tensión se muestran en la tabla 4, dando una visión general acerca de las diferentes metodologías para la implementación de estas en Alemania.

\section{GD en España}

Los sistemas fotovoltaicos solares en España se fundamentan en el autoabastecimiento de energía eléctrica y generación distribuida (CNE, Enero 2011), debido a que el marco regulatorio español ha evolucionado, comprendiendo el alto recurso energético que disponen (Ruiz , Colmenar, Gil, \& Molina, Mayo 2013); esto asegura niveles de rentabilidad sin ayuda, a corto y mediano plazos, denominado paridad de red (Ruiz, Colmenar, Gil, \& Molina, Mayo 2013).

La paridad de red significa que el costo de la generación de energía sin incentivos es el mismo que el precio de compra de energía de la red. Este punto es el instante en que los usuarios se vuelven indiferentes con respecto a la compra o a la generación de energía que consumen (Ruiz, Colmenar, Gil, \& Molina, Mayo 2013).

De acuerdo con el EPIA (European Photovoltaic Industry Association), los sistemas fotovoltaicos podrán competir en precio con las fuentes convencionales de generación en el año 2020 (EPIA, 2013).

El potencial más alto de España para aumentar la proporción de energías renovables en la generación de electricidad se encuentra principalmente en eólica y solar fotovoltaica (Ministry of Industry turism and trade, 2010b, 2010). La producción de energía fotovoltaica en España sigue muy de cerca el patrón de demanda de energía eléctrica durante

Tabla 4. Estrategias de investigación para incrementar la capacidad de introducción a la red de baja tensión de los sistemas fotovoltaicos y la implementación de su estatus en Alemania.

\begin{tabular}{ll}
\hline \multicolumn{1}{c}{ Estrategia } & \multicolumn{1}{c}{ Estatus de la implementación } \\
\hline Refuerzo de la red & $\begin{array}{l}\text { Statu Quo: Medidas para incrementar la potencia de corto circuito de la red } \\
\text { se emplean comúnmente en Alemania. }\end{array}$ \\
\hline $\begin{array}{l}\text { Limitación de potencia activa del alimentador a } \\
70 \% \text { de la capacidad instalada de los módulos } \\
\text { en la prueba de condición estándar }\end{array}$ & $\begin{array}{l}\text { con una capacidad instalada menor que } 30 \mathrm{kWp} \text { y sin la característica de ser } \\
\text { controlado remotamente. Desde 2012. }\end{array}$ \\
\hline $\begin{array}{l}\text { Potencia reactiva estática de provisión (caída } \\
\text { del control del factor de potencia) }\end{array}$ & $\begin{array}{l}\text { Statu Quo: Desde 2012 los usuarios son autorizados para demandar potencia } \\
\text { reactiva de los generadores conectados a la red de baja tensión. }\end{array}$ \\
\hline $\begin{array}{l}\text { Limitación automática de tensión por control } \\
\text { dinámico de potencia activa }\end{array}$ & $\begin{array}{l}\text { Futuro próximo: El control no está disponible en el comercio. Los códigos de } \\
\text { red necesitan revisiones futuras mediante investigación. }\end{array}$ \\
\hline $\begin{array}{l}\text { Limitación automática de tensión por control } \\
\text { dinámico de potencia activa/reactiva }\end{array}$ & $\begin{array}{l}\text { Futuro próximo: El control no está disponible en el comercio. Los códigos de } \\
\text { red necesitan revisiones futuras mediante investigación. }\end{array}$ \\
\hline $\begin{array}{l}\text { Transformadores de distribución con OLTC } \\
\text { (On-load Tap Changer) }\end{array}$ & Futuro próximo: Primeros prototipos están disponibles. \\
\hline
\end{tabular}

Fuente: (Stetz, Marten, \& Braun, 2013). 
todo el año y, por tanto, provoca relativamente menos problemas de integración a la red que los sistemas fotovoltaicos instalados en los países del norte de Europa (Cossent, Gómez, \& Olmos, 2011).

\section{GD en Japón}

En los últimos años Japón está introduciendo un nuevo concepto en el ámbito de la demanda para hacer frente en la alta penetración de la GD, la cual denominan área de demanda de un sistema autónomo de potencia. Este sistema adopta avanzados elementos de electrónica de potencia, así como alta inversión en tecnologías de comunicación, con el propósito de mantener la calidad de potencia y la seguridad de sistemas y así hacer uso eficiente de la energía, tanto para el usuario como el operador de red (Kobayashi \& Uemura, Development of Autonomus demand area power system in CRIEPI, 2003).

En 2008 se inició una investigación llamada TIPS o Triple I (Inteligent, Interavtive and Integrated) Power Systems. Esta investigación se desarrolla para mantener la calidad de potencia, la seguridad, estabilidad y confiabilidad de todo el sistema, además de contribuir a la reducción de gases de efecto invernadero, todo ello con el uso efectivo de la GD (Kobayashi \& Kurihara, Researche and development of grids integration distributed generation in Japan, 2009).

\section{GD en Estados Unidos}

El departamento de energía de Estados Unidos (DOE), estima que hay instaladas en el país más de 12 millones de unidades GD, con una capacidad agregada de 200 GW, la mayoría solamente proporcionan electricidad en situaciones de emergencia cuando la energía procedente de la red no está disponible (EIA Energy Information Administration, 2011). Incluso aunque los usuarios operan alrededor de $82.000 \mathrm{MW}$ de esta capacidad para funciones distintas a la potencia de apoyo, menos de $1 \%$ de los sistemas GD industriales se utilizan para producir electricidad que ayude a satisfacer los picos de demanda u operan de manera continuada (EIA Energy Information Administration, 2011).

Un estudio Ilevado a cabo por el Instituto Politécnico de Virginia y la Universidad Estatal de Virginia concluyó ( Pipattanasomporn \& Willingham, 2010):

- Las tecnologías de GD ofrecen ventajas comparativas respecto a las grandes plantas centralizadas en términos de eficiencia, fiabilidad y seguridad.

- En términos de eficiencia, la generación de energía próxima al lugar de consumo puede duplicar la eficiencia de las turbinas de gas de ciclo combinado y ofrece costos inferiores de capital y laborales.

- Algunos sistemas de GD son especialmente útiles para aplicaciones en donde pueda reciclarse energía de los residuos para proporcionar calor y frío a varias edificaciones.

- Tienen el potencial añadido de que la electricidad que producen es más fiable y segura en el caso de fallo del fluido eléctrico.

- Las tecnologías de GD permiten estabilizar la red y mejorar la fiabilidad del sistema en su conjunto.

- Debido a que los fabricantes de equipos pueden producir tecnologías de GD casi a cualquier escala, los operadores del sistema pueden situarla en cualquier sitio, y su modularidad significa que los generadores pueden adaptarse mejor a los pequeños incrementos de la demanda.

Los estados del sureste norteamericano (Arizona, California, Colorado, Nuevo México, Nevada, Texas y Utah), tienen el mayor potencial de energía solar del país, como se observa en la figura 4 . Se estima que el recurso de energía solar tenga un potencial anual de producción de 56 millones de GWh de energía generada a partir de sistemas fotovoltaicos o con energía solar concentrada (United States Congressional Research Service (CRS), 2009).

La tasa de crecimiento anual de generación de energía eléctrica a partir de sistemas fotovoltaicos fue de 30\% entre los años 2001 y 2010 (EIA Energy Information Administration, 2011). El elevado 
costo de la energía solar es el mayor desafío para la implementación de la GD a partir de este tipo de tecnologías. Por tal razón, DOE está financiando una iniciativa, conocida como el programa "SunShot", cuyo objetivo es reducir el costo de generación por energía solar a menos de 60 USD/ MWh (US Department of Energy (DOE), 2005).

\section{MARGO REGULATORIO DE LA GD}

Debido a las diferentes regulaciones gubernamentales, el rango de los generadores varía dependiendo del país. En el Reino Unido las plantas de GD con capacidades menores que $50 \mathrm{MW}$ no son despachadas en el mercado centralizado (Watson, 1999). La legislación sueca da tratamiento especial a los pequeños generadores con capacidad máxima hasta 1500 kW (Menges \& Barsantny, 1997;
VDEW, 1998; Swedish Electricity, 1997; Wijesinghe \& Lei Lai, 2011).

Las resoluciones que se encuentran en la legislación colombiana no tienen resaltadas las reglas o aspectos a tener en cuenta en el momento de hablar del concepto de GD. Sin embargo, existen ciertas definiciones que intentan introducir ideas relacionadas con esta temática, ya que algunos usuarios están empezando a emplear este tipo de tecnología para su propio uso. Dentro del marco regulatorio de Colombia se puede encontrar lo estipulado en las resoluciones CREG 084/96, CREG 085/96 y CREG 082/2002, las cuales hacen referencia a generación a pequeña escala, pero no ingresan en el marco que se tiene para generación distribuida (CREG, Comisión Reguladora de Energía y Gas, 1996; CREG, Comisión Reguladora de Energía y Gas, 2002).

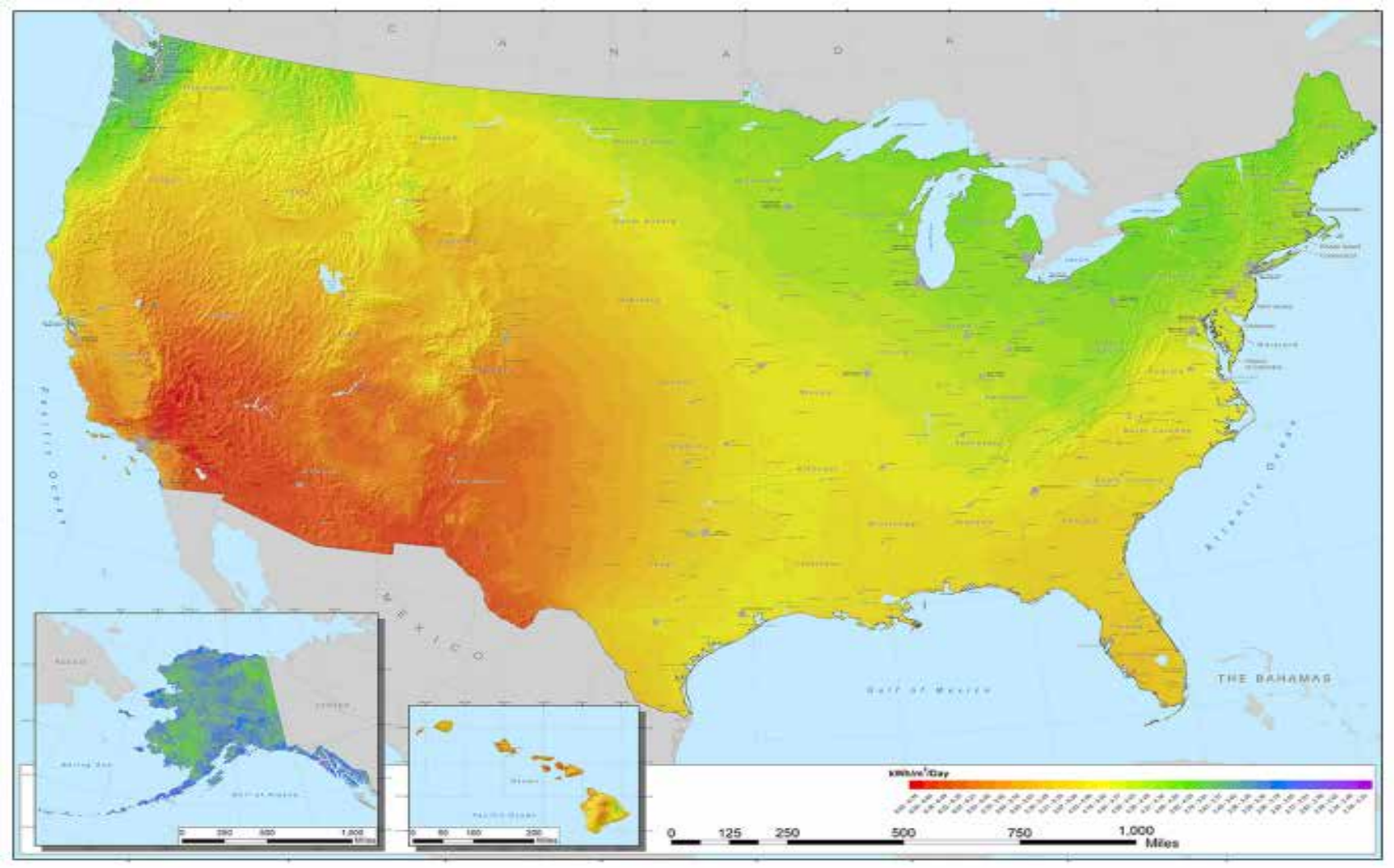

Figura 4. Recurso solar US.

Fuente: (NREL National Renewable Energy Laboratory, 2013). 
Como se encuentra definido dentro de la resolución CREG 084/96, se entiende por autogenerador aquella persona natural o jurídica que produce energía eléctrica exclusivamente para atender sus propias necesidades. Por tanto, no usa la red pública para fines distintos al de obtener respaldo del SIN, y puede o no ser el propietario del sistema de generación (CREG, Comisión Reguladora de Energía y Gas, 1996).

De igual forma dentro de la resolución CREG 085/96 se hace referencia a cogeneración cuando existe un proceso de producción combinada de energía eléctrica y energía térmica, que hace parte integrante de una actividad productiva, destinadas ambas al consumo propio o de terceros y destinadas a procesos industriales o comerciales (CREG, Comisión Reguladora de Energía y gas, 1996). Por tanto, cogenerador es aquella persona natural o jurídica que produce energía utilizando un proceso de cogeneración y que puede o no ser el propietario del sistema de cogeneración (CREG, Comisión Reguladora de Energía y gas, 1996).

A tenor de las definiciones dadas con anterioridad, se dice que un autoproductor corresponde a los autogeneradores y cogeneradores, como se enuncia en la resolución CREG 082/2002 (CREG, Comisión Reguladora de Energía y Gas, 2002). Como en Colombia no existe un esquema regulatorio para la aplicación de la generación distribuida, el concepto de autoproductor empieza a tomar bastante fuerza debido a que este es el único término que se encuentra en la reglamentación, siendo así el único instrumento al cual se acude en el momento de empezar a emplear este tipo de tecnologías.

Por otro lado, cuando se habla de la regulación sobre GD en el contexto internacional se debe mencionar países como Alemania o España, debido a que el primero se ha considerado como el país más avanzado en cuanto a generación mediante fuentes renovables, las cuales en muchos casos implican GD (Sepulveda, 2010). Un aspecto que tienen en común estos países es que no existe una definición oficial de GD, ni tampoco una regulación, estándar o norma que hable específicamente sobre el concepto de GD (Sepulveda, 2010).
En el caso español se tiene la regulación del nuevo sistema eléctrico, esta se fundamenta en la ley 54 de 1997 del sector eléctrico y su desarrollo normativo. En esta ley se incluyen esquemas de mercado en las actividades de generación y comercialización, además de la modificación del régimen especial. Cuando se modificó el régimen especial se agregó el tratamiento de residuos, biomasa, hidráulica, eólica, solar y cogeneración (Sepulveda, 2010). La mayor parte de las instalaciones de GD en España se acogen al régimen especial.

En el caso alemán cabe destacar que la nueva ley de energías renovables regula la adquisición, el transporte y la retribución de la electricidad producida mediante algunas tecnologías de GD, donde se fijan criterios de interconexión de las mismas. En cuanto a la cogeneración, esta viene regulada en forma independiente, con criterios técnicos y de interconexión propios (Bundesministerium Der Justiz, 2009).

En el caso de Norteamérica, a diferencia de Europa y Colombia, se está legislando directamente sobre GD, aunque se habla directamente de "Recursos Distribuidos de Energía (DER, Distributed Energy Resources). Cada estado establece su propia normatividad y reglamentación, manteniendo estándares federales en común, lo cual hace que el mapa de GD sea más complicado en este país. Para solucionar el dilema de la normatividad federal, el IEEE desarrolló en 2003 un estándar para conexión de GD (IEEE Guide for IEEE Std. 1547), el cual muestra cómo trabajar con este tipo de tecnologías avaladas normativamente. Un ejemplo de iniciativa estatal es el caso del estado de California, en donde se tienen tres organismos trabajando en torno a la normatividad de GD, los cuales son la California Public Utilities Comission (CPUC), la California Energy Comission (CEC) y la California Air Resources Board (CARB) (Ackerman, Andersson, \& Söder, 2001)". Estos organismos fomentan la GD a través de subsidios, incentivos para la inclusión de la misma en planes futuros de actuación. Asimismo, se incluye el sistema de medición neta, el cual permite al usuario medir la energía consumida menos la energía inyectada a la red (Grundi, 2008). 


\section{GESTIÓN DE LA DEMANDA}

La gestión de la demanda de energía (GDE) es un término ambiguo que ha sido interpretado de diversas formas en la regulación. Una definición restringida incluye todas las medidas que promuevan la reducción y/o el desplazamiento del consumo eléctrico final, mejorando la eficiencia en su utilización, pero sin modificar el contenido de los servicios finales (calor, iluminación, fuerza) que la energía eléctrica proporciona (Zeng, Liu, Wu, \& Nagan, 2011; Józef, Piotr, \& Mariusz, 2009) Sin embargo, es posible también contemplar medidas encaminadas a la adopción de nuevas pautas de consumo, que no han de significar necesariamente una pérdida de bienestar.

Ampliando el concepto con mayor detalle, se incluye el conjunto de acciones, ya sean impulsadas o realizadas por las empresas eléctricas, por otras empresas o instituciones o por la administración, cuyo objetivo común es influir sobre el uso que los consumidores hacen de la electricidad, de modo que se produzcan los cambios deseados, produciendo un ahorro de energía, así como el incremento de la eficiencia (Pérez, Sánchez, \& Pardo, 2005), bien sea en el ámbito de un solo usuario o en la curva de demanda que represente un grupo de estos.

El establecimiento de procedimientos que hagan posible la participación activa de la demanda por parte de los consumidores hace que no solo sean informados de los precios para que puedan reaccionar ante los mismos, sino que además contribuyan activamente a la formación de estos, además de poder proporcionar servicios al conjunto del sistema eléctrico con valor de mercado, como la interrupción en situaciones de emergencia, o para resolver congestiones, proveer reservas de operación y participar en la gestión de desvíos o de la compensación de energía reactiva (Pérez, y otros, 2007; Carmeli, Marco, Catellif, Marchegiani, \& Rosati, 2012).

Estos procedimientos se completan con otros que posibilitan al consumidor conocer el origen de la electricidad que consume, con lo que se permite la diferenciación de productos. De esta forma se proporciona a los consumidores una verdadera capacidad de elección en su suministro de electricidad, junto con las contribuciones a la eficiencia y a la mejora de la operación del sistema eléctrico.

Por ello, con la implementación de la GDE es posible obtener un ahorro energético y económico por la menor utilización de combustibles fósiles importados. Finalmente, a causa de la mayor eficiencia energética y de la menor utilización de combustibles fósiles, habrá menores emisiones de $\mathrm{CO}_{2}$ y de contaminantes atmosféricos.

\section{RECOMENDACIONES}

En este documento se reunieron los conceptos y aplicaciones que se han investigado en países como Estados Unidos, España, Alemania, Italia, Japón y Colombia, sobre la generación distribuida, especialmente en los sistemas fotovoltaicos. Asimismo, se mostraron distintos marcos regulatorios, donde algunos países impiden el desarrollo de este tipo de tecnología, o por el contrario, favorecen el avance de estas.

Por tanto, partiendo de todos los contenidos presentados en este documento, es preciso mencionar que para lograr adecuar e implementar los sistemas fotovoltaicos como alternativa de GD se debe trabajar con un sistema que permita entregar energía tanto al sistema de potencia como al usuario; conocido como SFVCR (Sistema Fotovoltaico Conectado a la Red).

Se recomienda trabajar con SFVCR, puesto que este permite ser adaptado en las futuras tecnologías que utilicen el concepto de GD aplicado a redes inteligentes, donde el usuario puede tener conocimiento de los eventos en tiempo real de su sistema de generación y del sistema de potencia, lo cual lo convierte en una parte activa de la gestión de la energía.

Por el alto costo de este tipo de tecnología, es pertinente realizar un estudio económico que muestre cómo se recuperaría la inversión inicial a mediano 
y largo plazos. De este modo es posible conocer la factibilidad que este tipo de aplicaciones puede bridar tanto al usuario como al sistema de potencia.

En aquellos países en donde no se cuenta con un marco regulatorio que no comprenda el uso masivo de generadores fotovoltaicos a pequeña escala, se recomienda que no exista inyección de energía a la red. Pero en el caso en que esto no se cumpla, los excedentes deberán ser mínimos, es decir, la generación debe ser cercana a la demanda. Esto permitirá que no existan discrepancias entre el operador de red y el usuario que tenga su propio sistema de generación a pequeña escala.

\section{FINANCIAMIENTO}

\section{Universidad Distrital Francisco José de Caldas}

\section{REFERENCIAS}

Ackermann, T.; Andersson, G., \& Söder, L. (2001). Distributed Generation: A definition. IEEE Electric Power System Researche, 57, 195-204.

Assi, A.; Jama, M., \& Al Kathairi, K. (2009). MATLABBased Modeling tool for designing, predicting and analyzing Grid Tied Photovoltaic Systems. ACTEA International conferences on:Advance in computational tools for engineering applications, 508-513.

Aste, N.; Adhikari, R., \& Del Pero, C. (2011). Photovoltaic Powered Distributed Generation Development in the Italian Context. Clean Electrical Power (ICCEP), International Conference on, 769-773.

Bayod, A. (2012). Future development of the electricity systems with distributed generation. Electric Power Systems Research, 84, 195-200.

Bundesministerium Der Justiz (2009). Ley de Energía Renovables. (Ministerio Federal Alemán de Justicia). Recuperado el 12 de noviembre de 2012, de: http://bundesrecht.juris.de/eeg_2009

Caamaño, M. (1998). Edificios fotovoltaicos conectados a la Red Eléctrica: Caracterización y Análisis. En Tesis doctoral. Madrid, España: Universidad Politécnica de Madrid, Escuela Técnica Superior de ingenieros de Telecomunicaciones.
Cardell, J., \& Tabors, R. (1998). Operation and control in a competitivemarket: distributed generation in a restructured industry. The Energy Journal Special Issue: Distributed Resources: Toward a New Paradigm of the Electricity Business, The International Association for Energy Economics, 111-135.

Carmeli, S.; Marco, M.; Catellif, Marchegiani, G., \& Rosati, D. (2012). Control Strategies and configuration on hybrid distributed generation systems. Renewable Energy, 41, 294-305.

Casmus, C., \& Eusebio, E. (2008). Micro-generation evalution of the zero emission technologies in the Portuguese market. Lisboa, Portugal: Instituto Superior de Engenharia.

CIGRE (1998). Impact of increasing contribution of dispersed generation on the power system; CIGRE Study Committee no 37 , Final Report. CIGRE.

CIRED (1999). Dispersed Generation; Preliminary Report of CIRED. Working Group WG04, 35-40.

CNE (enero 2011). Statistical information about the sales of electricity on special regime. December 2010. España: CNE.

Consorcio Energético CORPOEMA (diciembre, 2010). Plan de Desarrollo para las fuentes no convencionales de Energía en Colombia. Bogotá.

Cossent, R.; Gómez, T., \& Olmos, L. (2011). Large-Scale Integration of Renewable and Distributed Generation of Electricity in Spain: Current situation and Future needs. Energy Policy, 39, 8078-8087.

CREG, Comisión Reguladora de Energía y Gas. (1996). Resolución 084/96, Autogenerador conectado al sistema de interconexión nacional. Bogotá, Colombia: Ministerio de Minas y Energía.

CREG, Comisión Reguladora de Energía y gas (1996). Resolución 085/1996, Conegerador conectado al SIN. Bogotá: Ministerio de Minas y Energía.

CREG, Comisión Reguladora de Energía y Gas (2002). Resolución 082/2002, Cargos por uso de los sistemas de transmisión regional y distribución local. Bogotá, Colombia: Ministerio de Minas y Energía.

CREG, Comisión Reguladora de Energía y Gas (mayo, 1998). Resolución 070/98, Regalamento de 
Distribución de Energía Eléctrica. Bogotá, Colombia: Ministerio de Minas y Energía.

E.E.P.P.M. (febrero, 2008). Jepirachi wind farm-monitoring periods January 2004-July 2006. Medellín, Colombia.

EIA Energy Information Administration (2011). Annual Energy Outlook 2011, Report number: DOE/EIA0383 2011. Estados Unidos: EIA.

EPIA (2010). Solar Generation 6, 2010b. (EPIA) Recuperado el 11 de mayo de 2013, de: www.epia.org

EPIA (2013). European Photovoltaic Industry Association (EPIA). Recuperado el 01 de 06 de 2013, de: http://www.epia.org/about-us/about-epia/ mission-activities/

EPRI (2013). Distributed Electricity Resources (EPRI). Recuperado el 11 de mayo de 2013, de: http:// www.epri.com/Our-Work/Pages/Distributed-Electricity-Resources.aspx

Fathpour, S.; Tsia, K., \& Jalali, B. (2007). Two-photovoltaic effect in silicon. IEEE Journal of Quantum Electronics, 43(12).

Fundación de la Energía de la comunidad de Madrid (2012). Guía básica de la Generación distribuida. Madrid, España: Gráficas Elisa, S.A.

Gerol, W., \& Neudck (1990). The PN junction diode. En: Modular series on solid state devices (segunda ed.) Addison-Wesley Publishing Company.

Gischler, C., \& Janson, N. (noviembre 2011). Perspectivas sobre la generación distribuida mediante energías renovables en América Latina y el Caribe. Banco Interamericano de Desarrollo.

Grundi, P. (2008). Shell Energy Scenarios to 2050. pp. 8-10: Shell Internation BV.

GSE (2011). Aggiornamento del numero degli impianti e della potenza installata al 28 de Febbraio 2011 (GSE). Recuperado el 11 de mayo de 2013, de: www.gse.it

Hernández, A. (2012). Conference Electric Mobility. Bogotá, Colombia: [Conferencia], Seminar: Smart Grids on Electri Mobility.

IEA (2003). Potetial for Building integratged photovoltaiCS (IEA). Recuperado el 11 de mayo de 2013, de: www.iea-pvps.org
IEEE Guide for IEEE Std. 1547 (s.f.). IEEE Standard for interconecting distributed resourses with electric power systems. IEEE.

Institute, G. R. (1998). Distributed Power Generation: A Strategy for a Competitive Energy Industry. USA: Gas Research Institute.

International Energy Agency (2002). Distributed Generation in Liberalized Electricity Markets. New York.

Józef, P.; Piotr, B., \& Mariusz, K. (2009). Hybrid Power Systems-An Effective way of utilising primary energy sources. Renewable Energy, 34, 2414-2421.

Jurado, F.; Cano, A., \& Carpio, J. (2004). Biomass based on micro-turbine plant and distribution network stability. IEEE Energy Conversion and Management (45), 2713-2727.

Kobayashi, H., \& Kurihara, I. (2009). Researche and development of grids integration distributed generation in Japan. Power and Energy Society General Meeting 2009, 1-8.

Kobayashi, H., \& Uemura, S. (2003). Development of Autonomus demand area power system in CRIEPI. IFAC, Symposium on power plants and power system control 2003.

Lopes Ferreira, H.; Costescu, A.; L'Abbate, A.; Minneo, P., \& Fulli, G. (2011). Distributed Generation and Distribution Market Diversity in Europe. Energy Policy, 39, 5561-5571.

Menges, R., \& Barsantny, K. (1997). Die Liberalisierung der Strommärkte in Norwegen und Schweden. Zeitscltrift für Energiewirtschaft, Energiewirtschaftliches Institute an der Universität Köln, 21, 39-56.

Meyer, B.; Banberger, Y., \& Bel, I. (2010). Integration of distributed energy resources. IEEE POwer and Energy Society General Meeting.

Ministry of Industry, Turism and Trade (2010). National Action Plan on Renewable Energies in Spain (PANER) 2011-2020. España: Ministry of Industry, Turism and Trade.

Murillo, S., \& Roldan, P. (2012). Model of Application of Distributed Generation in Colombia Rural Zones. IEEE Transmission and distribution conferences and exposition (T\&D); Power and Energy Society, 1-9.

NREL, National Renewable Energy Laboratory (2013). United States Photovoltaic Solar Resource: Flat 
Plate Tilted at Latitude (NREL). Recuperado el 01 de 06 de 2013, de: http://www.nrel.gov/gis/images/ map_pv_national_hi-res_200.jpg

Ochoa, F. (2002). El sector eléctrico colombiano: orígenes, evolución y retos, un siglo de desarrollo (1882-1999). Interconexión Eléctrica Colombia S.A ESP (ISA).

Pepermansa, G.; Driesenb, J.; Haeseldonckxc, D.; Belmansc, R., \& D'haeseleerc, W. (2005). Distributed Generation: Definition, Benefits and Issues. Energy Policy, 33, 787-798.

Pérez, A.; Alfonso, D.; Álvarez, C.; Ibáñez, F.; Sánchez, C., \& Segura, L. (2007). Hybrid Biomass-wind Power Plant for Reliable energy Generation. Renewable Energy, 35, 1436-1443.

Pérez, J.; Sánchez, L., \& Pardo, M. (2005). La gestión de la demanda de electricidad. Fundación Alternativas, 1, 7, 8, 25.

Pipattanasomporn, M., \& Willingham, M. (2010). White Paper on "Distributed Generation". Virginia: Alexandria Research Institute, Virginia Tech, VA.

REN21 (2012). Renewable Energy Policy Report. Global Statutus Report 2011. pp. 1-80.

Rodríguez, H. (noviembre, 2008). Desarrollo de la Energía Solar en Colombia y sus perspectivas. Revista de ingeniería Universidad de los Andes (9), 83-100.

Ruiz , S.; Colmenar, A.; Gil, R., \& Molina , A. (mayo 2013). Distributed Generation: The Definitive Bost for Renewable Energy in Spain. Renewable Energy, 53, 354-364.

Sepúlveda, J. (2010). La generación distribuida en España. Tesis de pregrado. Madird, España: Universidad Carlos III de Madrid, Ingeniería Técnica Industrial-Electricidad.

Sharma, D., \& Bartels, R. (1998). Distributed electricity generation in competitive energy markets: a case study in Australia. The Energy Journal Special issue: Distributed Resources: Toward a New Paradigm of the Electricity Business, The International Association for Energy Economics, 17-40.

Singh, A. K., \& Parida, S. K. (diciemebre 16-19 2012). Need of distributed generation for sustainable develoment in coming future. International Conferences on Power Electronics, Drives and Energy Systems, 1-6.

Stetz, T.; Marten, F., \& Braun, M. (2013). Improved Low Voltage Grid-Integration of photovoltaic systems in Germany. Sustainable Energy, IEEE Transactions, 4(2), 534-542.

Strachan, N., \& Dowlatabadi, H. (2002). Distributed Generation and Distribution Utilities. Energy Policy.

Swedish Electricity (1997). SFS 1997:857. Swedish Electricity.

United States Congressional Research Service (CRS). (2009). Annual Energy Review 2009. Estados Unidos: CRS.

US Department of Energy (DOE). (2005). Basic Research Needs for Solar Energy Utilization: Report on the basic energy sciences workshop on solar energy utilization. Washington D.C: DOE.

VDEW (1998). Die Nordische Elektrizita"tswirtschaft im Wettbewerb, Bericht über die VDEW Studienreise nach Norwegen. Frankfurt, Alemania: VDEW.

Watson, J. (enero de 1999). Perspective of Decentralised Energy Systems in a liberalised Market: The UK Perspective. Rolf Wüstenhagen, Thomas Dyllick, St. Gallen, Institute for Wirtschaft und Ökologie (IWO)-Diskussionsbeiträge (72), 38-47.

Wijesinghe, A., \& Lei Lai, L. (2011). Small Hydro Power Plants Analyse and Development. Beijing, China: State Grid Energy Researche Institute.

Wizwlius, T. (1998). Series of Offshore Projects Planned. Wind Power Monthly, 14 (10), 23-24.

Zeng, J.; Liu, J.; Wu, J., \& Nagan, H. (2011). A multy-agent solution to energy management in hybrid renewable energy generation system. Renewable Energy, 36, 1352-1363.

Zuñiga, I., \& Botina, Y. (2012). Techincal Economic study for the implementation of autonomus photovoltaic system in the residential sector of Colombia. Transmission and Distribution: Latin America Conferences and exposition, 1-5.

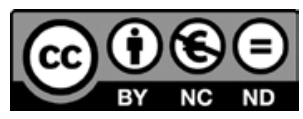

Tecnura • p-ISSN: 0123-921X • e-ISSN: 2248-7638• Edición especial 2014・pp. 157-172 